\title{
The heuristics of representation in science: the mechanisms and mathematical principles in physics of Descartes and Fermat
}

A heurística da representação na ciência: os mecanismos e os princípios matemáticos na física de Descartes e Fermat

\author{
Cássio C. Laranjeiras*1, Jojomar L. da Silva ${ }^{2}$, J.R.N. Chiappin ${ }^{3}$ \\ ${ }^{1}$ Instituto de Fisica, Universidade de Brasilia, Brasília, DF, Brasil \\ ${ }^{2}$ Departamento de Filosofia, Universidade de São Paulo, Brasil \\ ${ }^{3}$ Departamento de Economia, Universidade de São Paulo, São Paulo, SP, Brasil
}

Recebido em 30 de Janeiro, 2017. Revisado em 11 de Abril, 2017. Aceito em 12 de Abril, 2017.

\begin{abstract}
The objective of this article, which is part of the research program developed by the authors on the role of representation in science and science education, is to highlight the use of mechanisms and mathematical principles as representational heuristics of physical phenomena. They have been used by Descartes and Fermat in their respective investigations in the field of optics, more specifically in their analysis of the light refraction phenomenon. With examples drawn from the works of the cited authors, we sought to reveal the distinct, but not exclusive commitments and conceptions about the dynamics involved in building and developing scientific theories. From the heuristic point of view, we raise the hypothesis of the complementarity and convergence between both representations, with the argument it's up to the mechanisms to capture the constituting material principle of the phenomenon, while the abstract mathematical principles should take care of its formal organization. By emphasizing aspects related to the dynamics of the construction and development of scientific theories, heuristic elements essential to an understanding of the Nature of Science (NOS) and therefore relevant to the Descartes, Fermat, Epistemology of physics, Heuristics, Physics Education teaching and learning process of physics will emerge.
\end{abstract}

Keywords: Descartes, Fermat, Epistemology of physics, Heuristics, Physics Education

\begin{abstract}
O objetivo deste artigo, que faz parte de um programa de pesquisa desenvolvido pelos autores sobre o papel da representação na ciência e no ensino de ciências, é destacar o uso de mecanismos e princípios matemáticos como heurísticas representacionais dos fenômenos físicos e utilizada, respectivamente, por Descartes e Fermat em suas investigações no campo da óptica, mais especificamente em suas análises do fenômeno da refração da luz. Com exemplos extraídos da obra dos referidos autores, buscamos explicitar compromissos e concepções distintas, mas não excludentes, acerca da dinâmica de construção e desenvolvimento de teorias científicas. Do ponto de vista heurístico, levantamos a tese da complementaridade e convergência entre ambas as representações, sob o argumento de que caberia aos mecanismos capturar o princípio material constitutivo do fenômeno, enquanto os princípios matemáticos abstratos se encarregariam da sua organização formal. Ao enfatizar aspectos relacionados à dinâmica de construção e desenvolvimento de teorias científicas, elementos heurísticos essenciais a uma compreensão da Natureza da Ciência e, portanto, relevantes ao processo de ensino e aprendizagem da Física, surgirão.

Palavras-chave: Descartes, Fermat, Epistemologia da Física, Heurística, Ensino de Física.
\end{abstract}

\section{Introduction}

If we follow the analysis of Pierre Duhem (1861-1916), it's possible to identify in the history of science - and of Physics, in particular - two different paths through which one could treat natural phenomena based on the same empirical base: on the one hand, we can try to understand its real causes through causal explanations of empirical laws, on the other, we can employ abstract representations

*Endereço de correspondência: cassio@unb.br that seek to summarize and logically classify these same laws, without trying to explain them [1, p.7].

In the first path, which theoretical framework brings us back to the thinking of René Descartes (1596-1650), the causal explanations emerge initially associated with the use of mechanisms - established here as representative heuristic elements for solving problems in the context of a Cartesian program - to justify and operationalize the application of the mechanistic method. By representing geometry through algebra - giving rise to analytical ge- 
ometry - Descartes' work has established itself as a true milestone of representation in science, contributing to the development of an arsenal of resources to formulate and solve problems rationally.

In "La Dioptrique" (1637) 1] a treaty on optics where he formulates the law of sines for the refraction of light (in Discourses I and II, to be more specific), Descartes reveals and operationalizes his strategy for the "explication de la lumière et de ses rayons" [2, p.83], description/representation of the action of light through the laws of mechanical movement.

In the second path, the one of "abstract representations", we'll seek references originating in the heuristic resources used by Pierre de Fermat (1601- 1665), who employed specific mathematical principles to try to construct abstract theoretical models - based, for example, on his "principle of least time", a kind of union between Metaphysics and Geometry - as a strategy for the deduction/representation of empirical laws.

Throughout this article, which will highlight the use of mechanisms ${ }^{2}$ and mathematical principles as representational heuristics of Physics, more specifically in the field of Optics, we'll seek to explore how the "Research Programs" [4] of Descartes and Fermat - placed here at the origin of a broader heuristic program for representation in science - developed themselves, pointing out how they unfolded, their convergences and their repercussions.

\section{Descartes and the construction of mechanical models}

In Duhem's analysis, mechanistic physics was born in the 16th century with G. Galilei (1564-1642), M. Mersenne (1588-1648), B. Pascal (1623-1662) and C. Huygens (16291695), all contributing to its establishment and progress, together with the development of an associated methodology and philosophy. Descartes, however, would be the first to develop the Mechanism as a new conception of knowledge and science, looking to build a new intelligible framework of the world in which mechanical theory would play the role of unifying the structure of all science. To this end, Descartes articulated a theory of science to justify mechanistic physics as theoretical physics [5]. One of the central elements of this theory - which actually comprises a metaphysical, logic of science (methodology) and physical theory - is its concept of rationality, modeled on mathematics, in particular, on geometry. The justification of mechanistic theory as physical theory, in turn, is articulated through a system that not only turns metaphysics into a science, but also into a base to deduct and, consequently, to legitimize the principles of mecha-

\footnotetext{
${ }^{1}$ This is one of three scientific tests published by Descartes together with the "Discours de la Méthode". The other two tests that accompany it are "Les Météores" and "La Géometrie".

${ }^{2}$ According to Bunge "a mechanism is defined as what makes a concrete system tick, and it is argued that to propose an explanation proper is to exhibit a lawful mechanism" 3. p. 410].
}

nistic physics as causal principles of natural phenomena. Both the physical and metaphysical aim to offer causal explanations and are built using the same method. In this sense, Ontology creates a world of mechanical causes, Epistemology ensures the conditions to know with certainty and to grasp the essence and the cause of the things in this world, and Methodology, in turn, defines the nature and the means to build a physical theory and to solve problems [5] [6, pp.7-78].

In order to "fully eliminate the qualities of the study of material things" [7], Descartes lets go of substantial forms (qualities) as essential attributes of the body, retaining only its quantitative elements. His ontological theses about the separation between mind and body and about the quantitative attributes as essential properties of bodies, are presented not only to make the tangible world mathematical, but also to make it a pure effect of mechanical causes. As such, he seeks to institutionalize, from an ontological perspective, the Galilean dichotomy between primary and secondary qualities, turning the Aristotelian category of quality ${ }^{3}$ into the intellectual background on which he places a derived element in his system. Underlying this ontology, is a commitment to the idea that epistemology precedes ontology, which arises from the Cartesian acknowledgment of mathematics as the means to represent knowledge, and of the geometricdeductive method as the formal resource to organize it.

On these two epistemological assumptions, namely representation (algebraic symbols) and organization (mathematical and geometrical systems), rests the Cartesian criterion of rationality: the order of reasons. The more abstract and essential characteristic of what science is, is based on an idealization/abstraction of the geometricdeductive method. This abstraction aims to apply the criterion of the order of reasons (such as the rationality criterion) to fields that transcend mathematics and geometry. In Descartes' mind, the order according to which a discipline is organized is characterized as knowledge, which separates it from a revelation and an opinion.

Having established the problem of the nature of the physical theory, Descartes also tries to demonstrate that physical theories result in theoretical knowledge about the external world. The solution he presented to the problem concerning the objectivity of science is aligned with his conception of truth. The objects of our knowledge

\footnotetext{
${ }^{3}$ Aristotle's conception prevailed in the seventeen century, with his doctrine of substantial forms and of real qualities. According to this tradition, the qualities and powers constitute a category of being, and everything that causes sensible perception is assumed as a form of quality. As such, heat, color, soundness, solidity and numerous other properties are noticeable because the body in observation has certain powers. Cartesian ontology rejects not only the proliferation of beings, but also the category of real and independent qualities. In the opinion of Descartes, the only category is that of quantities. There is no place for qualities in the quantifiable world of cartesian science; they are disqualified as primary concepts as they are representable by algebra, i.e. they can't be interpreted as quantities.
} 
are representations in our mind. Without a direct access to the real world, how can we ensure the objectivity of our representations?

Through purely theoretical considerations about the nature of our faculties, of knowledge and of God, Descartes obtains an epistemological guarantee that the innate ideas are representations of real things in the world. This way, he ensures the application of the theory of the correspondence of truth with the basic mechanical concepts, which he considered as innate ideas. These concepts represent objects in the world. As such, the objectivity of the base spreads throughout the system through deduction. This Cartesian system of principles (common notions) ensures that deduction (collection of intuitions) leads from truth to truth, showing that the mechanical conceptual world, founded on causal explanations, is not only based on a possible representation of the world, but in a faithful representation of it.

From the Cartesian perspective, the application of an ideal axiomatic conception of science in the explanation of physical phenomena cannot be implemented if the first principles, or the connections between them and the empirical laws, are not known. In general, however, the understanding of such (necessary and essential) connections between the theoretical and empirical base, is not available. On the other hand, we should remember that Cartesian ontology makes extension the essential attribute of matter. This thesis makes an atomistic view of matter unfeasible. It follows that the Cartesian conception of science as an ideal axiomatic method cannot tolerate the employment of atomic hypotheses. To operationalize his theory on the coherence of truth, and even his ideal axiomatic system, Descartes decides, however, to employ hypotheses, even if only provisionally. This use seems to be a contingency of the implementation of his mechanical theory to explain physical phenomena, however, requiring an atomistic view of matter. His methodology employs this theory as a way to articulate and make effective the application of his mechanical theory. As such, the gap between the first principles and the empirical laws can be reduced through the hypostatization of a theory of matter to which mechanical properties are attributed.

In Descartes' mind, it's the use of science for practical purposes, where the "brevité de la vie" is a decisive factor, which requires the temporary employment of hypotheses to circumvent the infinite time required for the establishment of ideal science, that is, the deduction of all physics from metaphysics. This perspective clearly emerges early in Discourse I of "La Dioptrique", where he justified the use of hypotheses ["suppositions"] and analogies ["comparaisons"] in his "explanation of light and of its rays":

Now since my only reason for speaking of light here is to explain how its rays enter the eye, and how they may be deflected by the various bodies they encounter, I need not at- tempt to say what is its true nature. It will, I think, suffice if I use two or three comparisons in order to facilitate that conception of light which seems most suitable for explaining all those of its properties that we know through experience and then for deducing all the others that we cannot observe so easily. In this I am imitating the astronomers, whose assumptions are almost all false or uncertain, but who nevertheless draw many very true and certain consequences from them because they are related to various observations they have made. [2, v.6, p.83]

Pressed by the need to submit explanations articulated in science, this new structure of the being (extension, size and motion) sees the world as a machine, composed of inert bodies moved by physical necessity and unified by mechanical laws that are expressed precisely in terms of shape, size and motion. Cartesian science as a rigorous and thorough chain of reasons, starting from God until the particularity of the physical phenomena, remains an ideal that practical and provisional science is gradually approaching, however.

Using the example of blind man who uses the movements of a cane to perceive the objects around him [mechanical analogy], Descartes proposes a way in which light can be conceived as a "movement or an action". As he tells us:

In order to draw a comparasion from this, I would have you consider the light in bodies we call 'luminous' to be nothing other than a certain movement, or very rapid and lively action, which passes to our eyes through the medium of the air and other transparent bodies, just as the movement or resistance of the bodies encountered by a blind man passes to his hand by means of his stick [2, v.6, p.84].

Given that motion, from the Cartesian perspective, is the fundamental force (or power) of nature ${ }^{4}$ - lying at the root of all phenomena - an explanation of light and of its properties would involve knowing what kind of motion the action of light is. Hence the initial strategy of the mechanical analogy with the cane, which is complemented by a second analogy [of the "barrel of grapes"] in which Descartes introduces the idea of subtle matter [methodological employment of an atomic theory of matter], which, hypothetically, fills space and would allow him to explain light as an action that propagates in a straight line 5

\footnotetext{
${ }^{4}$ According to Sabra, this is simply a result of the metaphysical principle that movement is the only force that can be rationally stated to exist in nature 8 p.27].

${ }^{5}$ According to Sabra, "For although he [Descartes] does not conceive of the propagation of light as an actual movement, he still asserts that light is no more than a mechanical property of the medium
} 
Consider a wine-vat at harvest time, full to the brim with half-pressed grapes, in the bottom of which we have made one or two holes through which the unfermented wine can flow (see Figure 1). Now observe that, since there is no vacuum in nature (as nearly all philosophers acknowledge), and yet there are many pores in all the bodies we perceive around us (as experience can show quite clearly), it is necessary that these pores be filled with some very subtle and very fluid matter, which extends without interruption from the heavenly bodies to us.

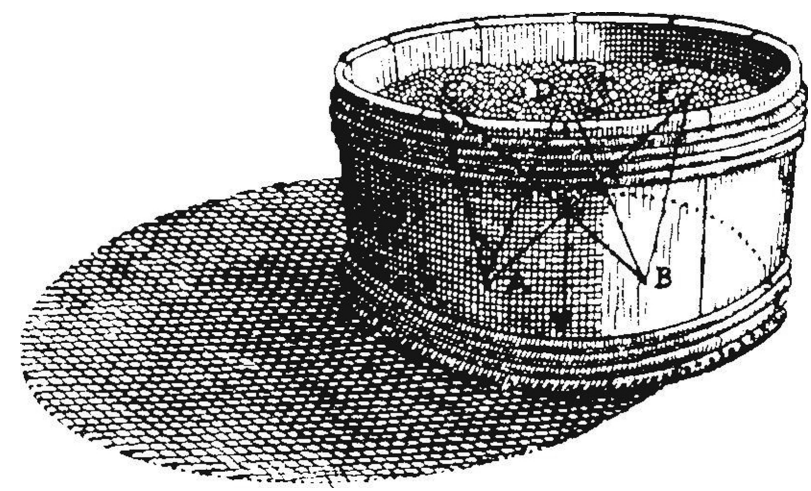

Figure 1: Figure extracted from the original text. See ref. [2, v.6, pp.86-87]

Now, if you compare this subtle matter with the wine in the vat, and compare the less fluid or coarse parts of the air and other transparent bodies with the bunches of grapes which are mixed in the wine, you will readily understand the following. The parts of wine at one place tend to go down in a straight line through one hole, while the parts at other places also tend at the same time to go down through these two holes, without these actions being impeded by each or by the resistance of the bunches of grapes in the vat. (...) In the same way, all the parts of the subtle matter in contact with the side of the sun facing us tend in a straight line towards our eyes at the very instant they are opened, without these parts impeding each other, and even without their being impeded by the coarse parts of the transparent bodies which lie between them. (...) In the same way, considering that the light of a luminous body must be regarded as being not so much its movement as its action, you must think of the rays of light as nothing other than the lines along which this action tends [2, v.6, pp.86-87].

transmitting it; it is, as we have seen, a static pressure existing simultaneously in all parts of the subtle matter that pervades all space [8, pp.78-79].
As a consequence of the practical impossibility of fully developing an ideal science, and in order to improve our knowledge of natural phenomena, he postulates materials systems of defined corpuscles, whose motion, size, shape and mechanical action are responsible for the production of all phenomena. The straight propagation of light would therefore be subject to deviations or buffering in the interaction with other bodies, in the same way that the movement of a ball, or of a stone thrown in the air, is cushioned by bodies that it finds on its way. In this sense, light, conceived as action, should follow the same laws of mechanical motion. This is clearly explained when Descartes compares the luminous ray to a ball put in motion by a racket, and presents us with his characterization mechanism of the refraction phenomenon. Let's read his own words:

Consider that, if a moving ball encounters obliquely the surface of a liquid body through which it can pass more or less easily than through that which it is leaving, it is deflected and changes its course when it enters:

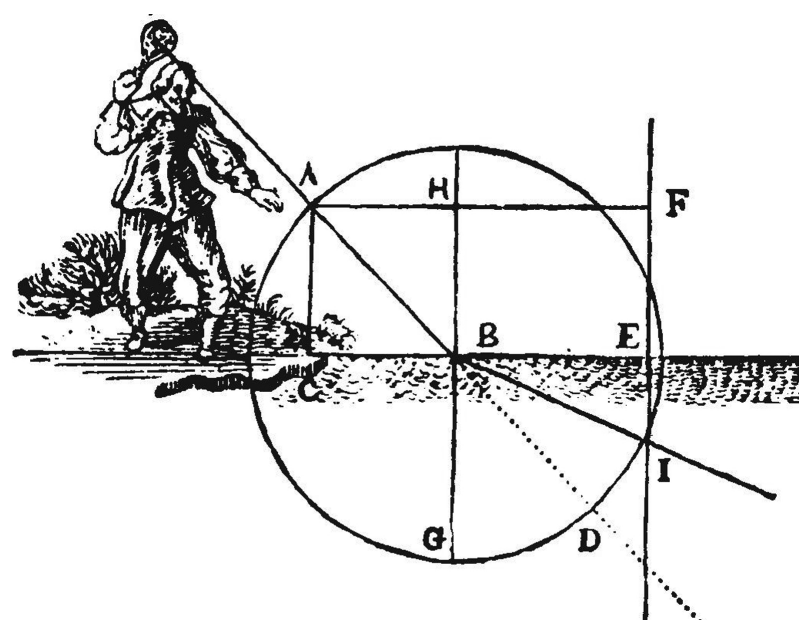

Figure 2: Figure extracted from the original text. See ref. 2. v.6, pp.91-93]

as, for example, if, being in the air at point $\mathrm{A}$ (fig. 2), it is impelled towards B, it will indeed go in a straight line from $\mathrm{A}$ to $\mathrm{B}$, if neither its weight nor some other particular cause prevent it; but, at point B, where I suppose it to encounter the surface of the water CBE, it is deflected and takes a path towards I, going moreover in a straight line from $\mathrm{B}$ to $\mathrm{I}$, as is easy to verify by experiment(...) Finally, consider that the rays are also deflected, in the same way as the ball just described, when they fall obliquely on the surface of a transparent body and penetrate this body more or less easily than the body from which they come. This mode of deflection is called 'refraction'. 2, v.6, pp.91-93] 
In Descartes, a tension can be clearly seen between two concepts of physical theory. On the one hand, a theory is conceived as an ideal axiomatic system, based on well-founded concepts and on self-evident principles; i.e., all physical phenomena should, in principle, be deducted from such concepts and principles. In this sense, his conception of physical theory emerges here, or as Sabra states: "as a deductive system whose premises are rooted in metaphysics" [8, p.27]. On the other hand, this theory is a definition that, through the use of figurative representations, analogies or models, establishes the bonds between the conceptual mechanic system and the empirical base itself. Such mechanical models are based on defined and concrete mechanisms of matter in motion, instead of employing a rigorous chain of logical reasons 6 In addition, they have the status of hypotheses, and not of self-evident presuppositions.

Still in "La Dioptrica", Discourse II, using the research strategies established in Regulae, more specifically Rules $\mathrm{V}$ and VI, Descartes gives us an explanation of the phenomenon of reflection as a type of intermediate step for the understanding of what was to come next, namely the exact determination of what he coined as being the "quantity" of the refraction 7. To this end, he replaces the surface CBE (thought of as a liquid surface in the previous example - characterization of refraction) by a rigid surface. His mechanism is presented in the following way:

Let us suppose that a ball impelled by a tennis racquet from $\mathrm{A}$ to $\mathrm{B}$ meets at point $\mathrm{B}$ the surface of the ground CBE (fig. 3), which stops its further passage and causes it to be deflected; and let us see in what direction it will go. To avoid getting involved in new difficulties, let us assume that the ground is perfectly flat and hard, and that the ball always travels at a constant speed, both in its downward passage and in rebounding, leaving aside entirely the question of the power which continues to move it when it is no longer in contact with the racquet, and without considering any effect of its weight, size or shape.

(...) Moreover, it must be noted that not only the determination to move in a certain direction but also the motion itself, and in general any sort of quantity, can be divided into all the parts of which we can imagine that it is composed. And we can easily imagine that

\footnotetext{
${ }^{6}$ The role of hypotheses and analogies in the [deductive] Cartesian explanation scheme is clearly pointed out in his "Rules for the Direction of the Mind" (1628), more specifically in rule VIII, where he advises the investigator against the insistence on the strict application of the preceding rules when the nature of the problem so requires.

${ }^{7}$ Refraction is treated as a special case of reflection, a case in which the perpendicular component of velocity is changed by the surface CBE.
}

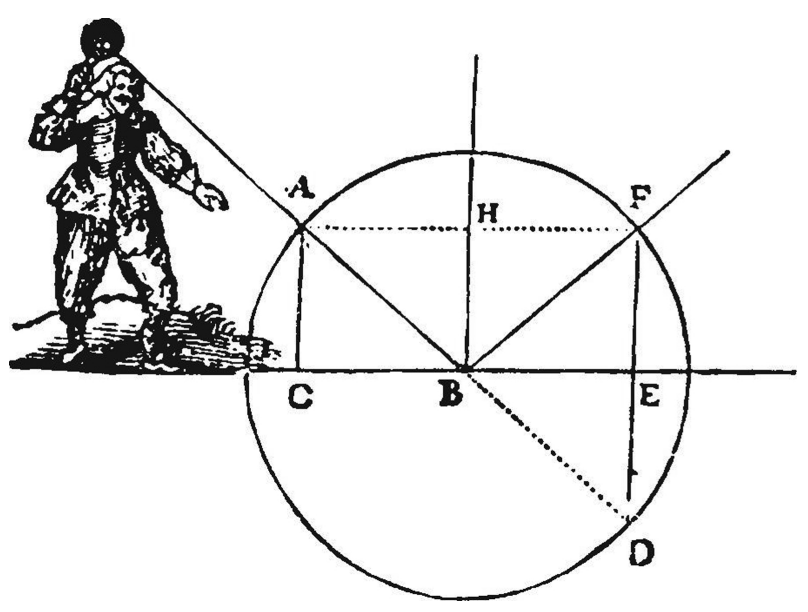

Figure 3: Figure extracted from the original text. See ref. 2 v.6, pp.93-95]
the determination of the ball to move from A towards B is composed of two others, one making it descend from line AF towards line $\mathrm{CE}$ and the other making it at the same time go from the left $\mathrm{AC}$ towards the right $\mathrm{FE}$, so that these two determinations joined to- gether direct it to $\mathrm{B}$ along the straight line AB. And then it is easy to understand that its encounter with the ground can prevent only one of these two determinations, leaving the other quite unaffected. For it must indeed pre- vent the one which made the ball descend from AF towards CE, because the ground occupies all the space below CE. 2, v.6, pp.93-95, em- phasis added]

Here, it's worth emphasizing the Cartesian strategy of splitting the "determination" 8 of the ball movement [understood as its speed] toward $\mathrm{AB}$ into two [more simple] parts, namely into the direction AC (vertical component) and into the other direction AF (horizontal component). This strategy, as we will see later, will be strongly criticized by Fermat. In his Rule V ("Regulae"), Descartes made it clear that complicated and obscure propositions should be gradually reduced to more simple propositions, seeking a more adequate understanding of the former based on the latter.

By describing the route the ball would take after its collision with the surface CBE, based on geometrical arguments and always considering its speed module as unchanged, Descartes builds a deduction of the exact point that the ball will reach. Let's see how he does this, following the figure of the previous citation:

\footnotetext{
${ }^{8}$ It is important to note that Descartes distinguishes between what he calls 'force' of the ball movement and its 'determination'. Our understanding is that when referring to the 'force', he has the speed module in mind, and from what he says in "determination", he thinks of speed as a vector (it may be divided into its components), superposing this last concept a few times over amount of motion.
} 
To discover in precisely what direction the ball must rebound, let us describe a circle, with its centre at $\mathrm{B}$, which passes through point $A$; and let us say that in as much time as the ball will take to move from $\mathrm{A}$ to $\mathrm{B}$, it must inevitably return from $\mathrm{B}$ to a certain point on the circumference of the circle. This holds in so far as the circumference contains all the points which are as far the circumference contains all the points which are as far from $\mathrm{B}$ as $\mathrm{A}$ is, and the ball is supposed to be moving always at a constant speed. Next, in order to determine precisely to which point on the circumference the ball must return, let us draw three straight lines $\mathrm{AC}, \mathrm{HB}$, and FE, perpendicular to $\mathrm{CE}$, so that the distance between $\mathrm{AC}$ and $\mathrm{HB}$ is neither greater nor less than that between $\mathrm{HB}$ and FE. And let us say that in as much time as the ball took to move towards the right side from $\mathrm{A}$ (one of the points on the line $\mathrm{AC}$ ) to $\mathrm{B}$ (one of those on the line $\mathrm{HB}$ ), it must also advance from the line HB to some point on the line FE. For all the points on the line FE are equidistant from the corresponding points on $\mathrm{HB}$, as are those on line $\mathrm{AC}$; and also the ball is as much determined to advance towards taht side as it was before. So it is that the ball cannot arrive simultaneously both at some point on the line $\mathrm{FE}$ and at some point on the circumference of the circle AFD, unless this point is either D or F, as these are the only two points where the circumference and the line intersect. Accordingly, since the ground prevents the ball from passing towards $\mathrm{D}$, it is necessary to conclude that it must inevitably go towards F. And so you can easily see how reflection takes place, namely at an angle always equal to the one we call the angle of incidence. In the same way, if a light-ray coming from point $\mathrm{A}$ falls at point $\mathrm{B}$ on the surface of a flat mirror CBE, it is reflected towards $\mathrm{F}$ in such manner that the angle of reflection $\mathrm{FBE}$ is neither greater nor less than the angle of incidence ABC. [2, v.6, p.96]

Note that, in his deduction of the equality of the angles of incidence and reflection, Descartes assumes that the reflective surface does not alter the speed module of the ball, i.e. the speed module of the incidence is equal to the speed module of the reflection. This would correspond to a perfectly elastic collision, where the kinetic energy of the system remains unchanged ${ }^{9}$

\footnotetext{
$\overline{{ }^{9} \text { Sabra calls our attention }}$ to the fact that Descartes makes no reference to the perpendicular component of incident velocity, not assuming, for example that it will be reverted by the reflective surface [8 p.85].
}

Following this chain of geometric reasoning, Descartes returns to the situation where the ball encounters a liquid surface, assuming this time that this surface will causing a deviation in the trajectory of the ball [his analogy for the refraction of light process], which will increase as the inclination with which the ball hits the surface increases, reaching a situation in which the ball will suffer reflection and not refraction.

And we may note here that the deflection of the ball by the surface of the water or the sheet is greater, the more oblique the angle at which it encounters it, so that if it encounters it at a right angle (as when it is impelled from $\mathrm{H}$ towards B) it must pass beyond in a straight line towards $\mathrm{G}$ without being deflected at all [fig. 4].

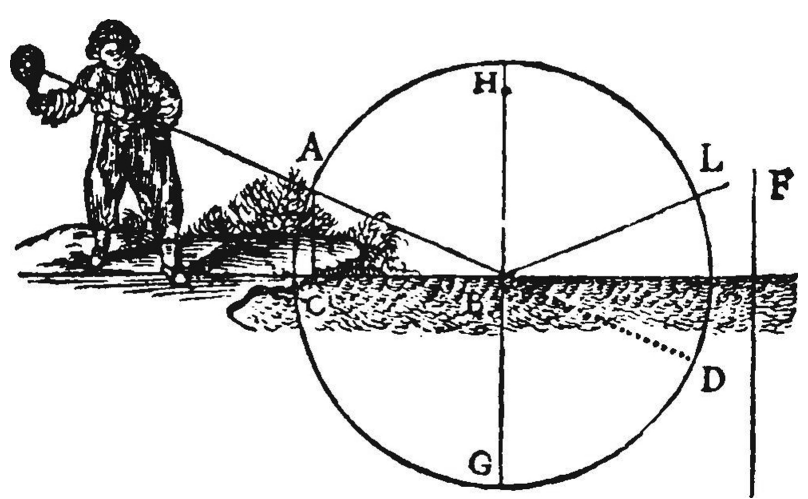

Figure 4: Figure extracted from the original text. See ref. 2, v.6, p.99]

But if it is impelled along a line such as AB, which is so sharply inclined to the surface of the water or sheet CBE that the line FE (drawn as before) does not intersect the circle $\mathrm{AD}$, the ball ought not to penetrate it at all, but ought to rebound from its surface B towards the air L, in the same way as if it had strick the earth at that point [2, v.6, p.99].

While noting that no refraction occurs in the case of a normal incidence (HB), and that the incident and refracted ray turn in the same direction, thus revealing to us the phenomenon of total reflection - in which case the line FE does not cut the circle AD - he doesn't consider the critical intermediate case, which occurs when FE is tangential to the circle. In this case, the angle of incidence is such that the refracted ray makes 90 degrees with the normal. After presenting his explanation for reflection, Descartes now seeks to determine the exact "quantity" of refraction suffered by ball that hits a liquid surface in such a way that it passes through the surface with its velocity increased by one-third in relation to the air.

But let us make yet another assumption here, and suppose that the ball, having been first 
impelled from $\mathrm{A}$ to $\mathrm{B}$, is again impelled at point $\mathrm{B}$ by the racquet $\mathrm{CBE}$ [fig.5] which increases the force of its motion, say by a third, so that it can then make as much headway in two seconds as it previously made in three. This will have the same effect as if the ball were to meet at point $\mathrm{B}$ a body of such nature that it could pass through its surface CBE, one-third again more easily than through the air. And it follows manifestly from what has already been demonstrated that if you describe the circle $\mathrm{AD}$ as before, and the lines $\mathrm{AC}, \mathrm{HB}, \mathrm{FE}$ so that there is a third less distance between FE and $\mathrm{HB}$ than between $\mathrm{HB}$ and $\mathrm{AC}$, then point I, where the straight line $\mathrm{FE}$ and the circular line AD intesect, will indicate the position towards which the ball must be deflected when at point B.

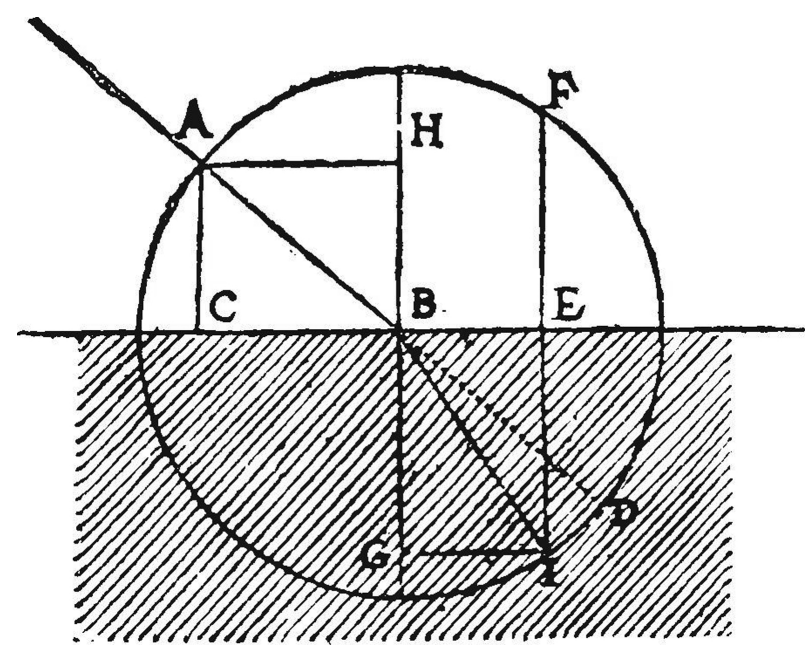

Figure 5: Figure extracted from the original text. See ref. 2, v.6, p.100]

Now we can also draw the converse of this conclusion and say that since the ball which comes in a straight line from A to B is deflected when at point $\mathrm{B}$ and moves on towards $\mathrm{I}$, this means that the force or ease with which it penetrates the body CBEI is related to that with which it leaves the body ACBE as the distance between $\mathrm{AC}$ and $\mathrm{HB}$ is related to that between $\mathrm{HB}$ and $\mathrm{FI}$ - that is, as the line $\mathrm{CB}$ is to BE. 2, v.6, p.100]

According to Descartes' reasoning, the direction of refraction is determined by the point I at which FE cuts the circle. The trajectory of the refracted ray is determined by the relation $B E=\frac{2}{3} C B$, and the ratio between the speed of the angle of refraction $\left(v_{r}\right)$ - ['force' (speed module) with which the ball enters the body CBEI (water)] - and the speed of the angle of incidence $\left(v_{i}\right)$ ['force'(speed module) with which the ball leaves the body ACBE (air)] - is given by $\frac{v_{r}}{v_{i}}=\frac{C B}{B E}$. The last relationship, not only matches the statement that the speed of light is a property of the environment in which it travels 10 , it also clearly expresses the idea that the sines of the angles are in an inverse ratio of their respective velocities, i.e., $\frac{\sin i}{\sin r}=\frac{v_{r}}{v_{i}}=n$, where $\mathrm{n}$ is a constant 11 .

The idea of an ideal axiomatic theory of physics is essential to rationally explain the objectivity of science (theoretical knowledge), in addition to Descartes' commitment to the objective (metaphysical) value of effective physical theories. Such theories do not share the conditions required by the ideal physical theory, since they employ hypotheses as intermediate chains. As such, this question immediately arises: how to explain the theoretical knowledge produced by them? This theoretical knowledge is established through the relation between these (effective) theories and the ideal axiomatic system that represents the world. Descartes seems to understand that the objective value (theoretical knowledge) of effective physical theories is defined in terms of their contribution to bringing them closer to the ideal physical theory, i.e. to the very understanding of this latest theory. The increase in our understanding of empirical laws and the relations between such laws and the conceptual mechanic system, decreases the variety of alternative hypotheses that connect self-evident principles to the empirical base. This leads to a broadening of our knowledge of the (real) essential and necessary relationships between these two bases, which constitutes the objective value of effective physical theories. Its practical value is given through its application to nature, constituting a relation between physical theory and the world of phenomena.

\section{Fermat and the use of mathematical principles}

In September 1637, soon after coming into contact with Descartes' 'La Dioptrique' through Marin Mersenne 12 , Fermat sent the latter a letter expressing his reservations in relation to the arguments presented by Descartes in his proof of the laws of reflection and refraction. In this correspondence, which ended up being the first of a sequence of letters, Fermat questioned the idea that the effects of light should be understood based on our knowledge about motion. In addition, he drew attention to what he called a 'particular disagreement' ['particuliére disconvenance'] in Descartes' reasoning 9 , p. 109], by not confronting his mechanical analogy of the ball's motion,

${ }^{10}$ According to Sabra, this idea had already been used by Latin writers since the translation of Ibn al-Haythan's 'Book of Optics', and is already found in Ptolemy. When Descartes formulated it in 1619-21, he departed from the generally accepted view that speed is greater in more dense means.

${ }^{11}$ This result will be left as a legacy to corpuscular theory, that would adopt it through a similar proof shown by Newton 8 p.111]. ${ }^{12}$ At the time, Mersenne played an important role in the promotion of scientific ideas in Europe, facilitating the contact between various scientists, and he ended up working as intermediary between Descartes and Fermat, who exchanged several letters through Mersenne. 
affected by a succession of small movements, with the idea of the instantaneity of the propagation of light 13 .

Fermat said he couldn't accept Descartes' reasoning, which he considered logically flawed as legitimate proofs and demonstrations of either reflection or refraction 9 , P.109]. For Fermat, Descartes's procedure was arbitrary because he had chosen a system of reference by presupposing the result that he wished to obtain in advance and which he considered valid [9, P.110].

In a letter written in 1664 to an unknown recipient, he even refers to the Cartesian proportions, used as the foundation for his 'La Dioptrique', as a true fallacy 14 His distrust was based on three basic arguments, namely that analogies cannot establish evidence, that the assumption that light has greater speed in more dense means contradicts common sense ${ }^{15}$, and, finally, that the assumption that one of the directions or 'determinations' of ball motion must remain unchanged after contact with the medium, cannot be justified [10, p. 485-486] 16

To justify his assumption about the conservation of the horizontal component on the reflecting surface (a real and impenetrable surface) Descartes had relied on the fact that the surface did not oppose the movement of the ball in that direction $\left[9\right.$, p.114] ${ }^{17}$

To deduct the Law of Refraction, Fermat made use of his own method, the "method of maxima and minima" 18 , which he had invented eight years before starting (1637) his correspondence around the work of Descartes. In this method, based on the metaphysical principle that nature

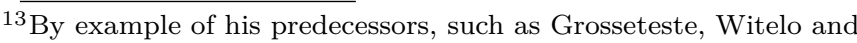
Kepler, Descartes subscribed to the doctrine of the instantaneous propagation of light.

14'Sa démonstration me sembla un véritable paralogisme.' 10 p. 485]

${ }^{15}$ Fermat worked with the idea that the speed of light is smaller in denser media (or more refringent) than air. Martins and Silva remind us that at this time it was not known how to measure the speed of light in a refractive medium, which prevented the decision of whether the speed was greater or less in these means 22 p.457, footnote].The assuption that light has greater speed in denser media has been adopted by Descartes since his earliest studies of optics (1616-1621). The idea was not new and has its origins in the works of Ptolemy (100-170 AD) and Ibn al-Haythan (965-1040 AD) in Optics.

${ }^{16}$ According to Sabra, if the first reason is removed, it would be wrong to believe the last two would really be among the reasons for the rejection by Fermat of Descartes' proof. The foundation of his argument lies in the fact that the question of the speed of light had never been the object of discussion in the correspondence between the two, and that Fermat's objection regarding Descartes' horizontal component of the 'determination of motion' appears for the first time only in 1658, in a letter to Clerselier, i.e. in the second phase of his discussion about ' $L a$ Dioptrique' 8 p. 117].

${ }^{17}$ According to Sabra "in agreement with a certain a priori conception of matter, and in accordance with the decision to deal with natural phenomena in a purely mathematical fashion, Descartes tried to deduce the reflection law from merely assuming the impenetrability of matter and the conservation of the absolute quantity of motion 8 . p. 92]

${ }^{18}$ This method was described by Fermat in the treaty "Méthode Pour la Recherche du Maximum et du Minimum", originally written in Latin ("Methodus ad Disquirendam Maximam et Minimam"), and sent, via Mersenne, to Descartes, who received it in the beginning of 1638 .

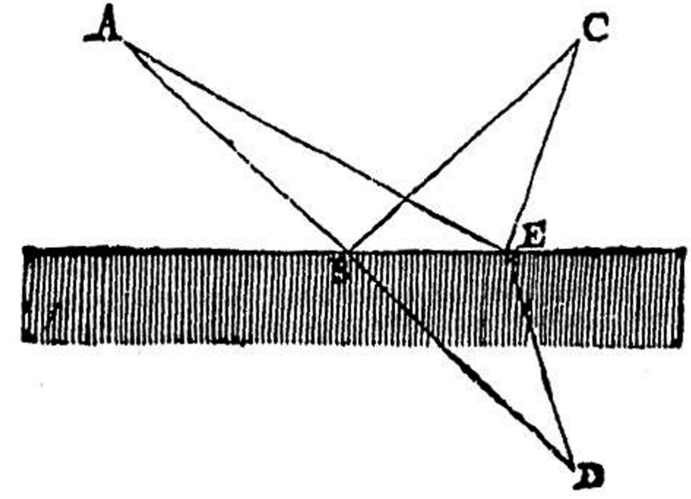

Figure 6: Figure utilized by La Chambre for his demonstration. Extracted from the original text. See ref. [2 v.6, p.328]

performs its actions in the most simple and economical way [optimization principle], the principle of least time is presented.

In 1657 , Fermat had received a copy of a treaty on light [La Lumière], which had been sent to him by the author himself [the physician Marin Cureau de la Chambre] and published that same year, where light [able to move instantaneously] was presented as a quality and not as a substance [11. In this sense, reflection and refraction were explained based on what was described as the 'animosity' and 'natural antipathy' between light and matter.

Although not convinced by the arguments presented by La Chambre in his treaty, Fermat made a point of emphasizing a common aspect between the two in a thank-you letter [august 1657], namely the principle that nature always acts by choosing shortest path 19

In order for us to better understand the path adopted by Fermat in the development of his principle of refraction - formulated based on the "principle of least time" let us quickly review a problem that was presented by La Chambre in the aforementioned Treaty, where he, making reference to the successful implementation of the principle of economy [that nature always operates along the shortest path] to the reflection of light on flat surfaces, presented a geometric demonstration in which, assuming that the angle of incidence is equal to the angle of reflection, the path followed by an incident ray at point B (starting from A) and reflected until C, is necessarily shorter than the one incident at point $\mathrm{E}$ (starting from A) and also reflected until C. The following figure 6 was used by La Chambre for his demonstration.

The geometric demonstration is made by extending the line $\mathrm{AB}$ until $\mathrm{D}$, making an extension of the same length of the reflected ray $\mathrm{BC}$, such that $\mathrm{ABD}$ is equal to $\mathrm{ABC}$, and also extending the line $\mathrm{AE}$ until $\mathrm{D}$, with its extension having the same length as EV, such that $\mathrm{AED}$ is equal to AEC. As such, one can see that $\mathrm{ABD}$ is shorter than AED (AE+ED), because together they form

\footnotetext{
19 "First, I acknowledge with you the truth of this principle: that nature always operates along the shortest path." [12, p.354].
} 
a triangle with $\mathrm{ABD}$ as its base and $\mathrm{AE}$ and $\mathrm{ED}$ as the two sides, since in any triangle, two sides taken together are longer than the third. Through this reasoning, therefore, it was clear to La Chambre that the path ABC, assuming equality between the incidence and reflection angles, was necessarily shorter than AEC.

Right after this demonstration, La Chambre makes reference to two difficulties that he identified in the path to the effective adoption of this principle [of the shortest way]. This is exactly where the problem arises which we mentioned previously. Let us see how he presents the first of these difficulties in his own words:

There are reflections that are made in concave mirrors, where the angles are equal, but the lines are always greater than if they were different (see Figure 7).

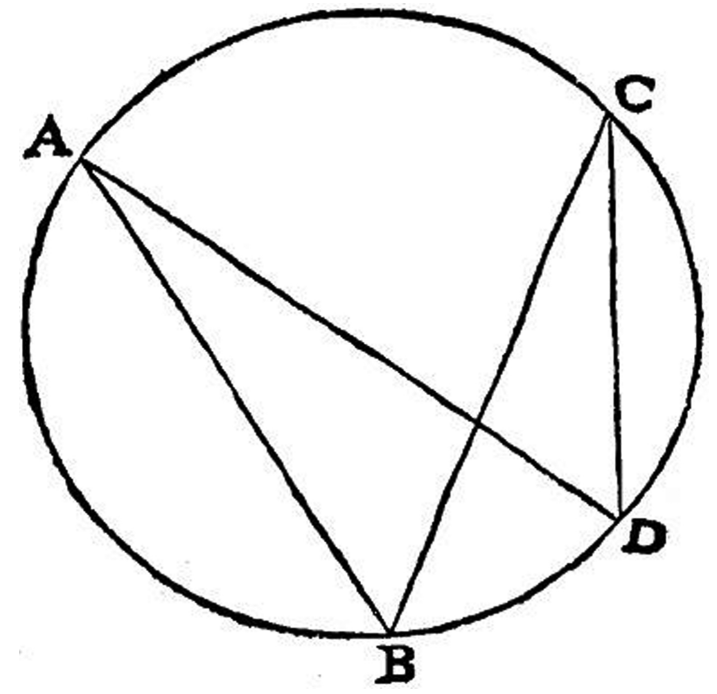

Figure 7: Figure extracted from the original text. See ref. 11. pp. 313-314].

The lines $\mathrm{AB}$ and $\mathrm{BC}$, which form equal angles, are larger than the lines $\mathrm{AD}$ and $\mathrm{DC}$, which form different angles, as can be demonstrated by geometry. Therefore, there is a general rule that the equality of the angles comes from shorter lines [11, pp. 313-314].

The second difficulty concerned the behavior of light in refraction.

If nature makes its movements through the shortest lines, it should necessarily also do this in refraction. In any case, the lines that contain the incidence and refraction angles are larger than those $[\mathrm{ADC}]$ outlined from one end to the other (see Figure 8).

Since the $\mathrm{AB}$ and $\mathrm{BC}$ lines, made by the refraction, are larger than $\mathrm{AD}$ and $\mathrm{DC}$, given that $\mathrm{AB}$ and $\mathrm{BC}$ form two sides of a triangle, with ADC as its base, and two sides are larger

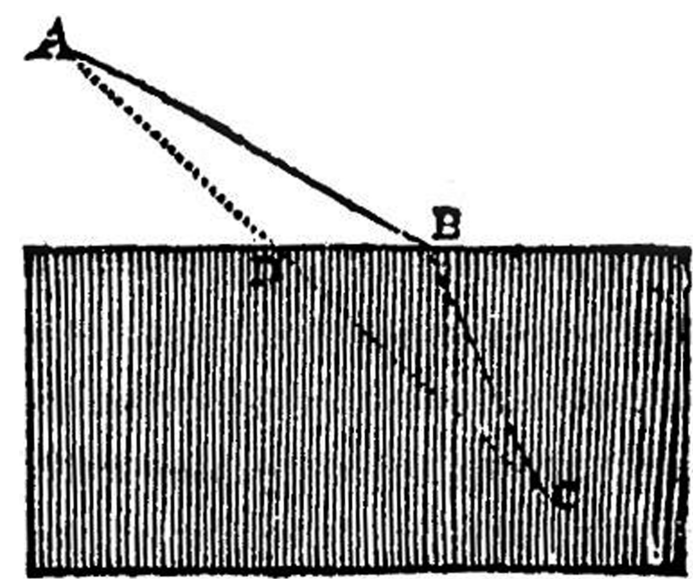

Figure 8: Figure extracted from the original text. See ref. 11, pp. 314-315].

than the third side considered in itself. 11, pp. 314-315].

In response to these difficulties, La Chambre argued that in the cases in question [in refraction], light is not "free" to move, and is subject to constraints that force it to follow a different path instead of the one it would follow if it were moving according to its "natural inclination" 11 . p. 323]. In this sense, the principle of the shortest path should be understood as ruling not the actual behavior of light, but its propensity, effectively expressed solely in the case of reflection on flat surfaces [8, p. 139].

Fermat tried to overcome these difficulties by looking at them from a different perspective, adopting the idea that "the principle of physics is that nature performs its movements through the most simple paths" [12, p.355]. Assuming that different media offer different resistances, and that there is always a defined proportion between these two resistances [12, p.356], Fermat worked with the idea that the straight line joining two points in such means may not be the path that minimizes resistance, and therefore not the simplest path. Fermat recasts the problem taking the figure used by La Chambre as reference (see figure of the citation above), where a surface DB separates media of different resistances to the passage of light - with the medium of incidence (which contains point $\mathrm{C}$ ) having half the resistance of the medium of refraction (which contains point A). In this sense, Fermat explains, the sum of the resistances along the incident ray $\mathrm{CB}$ and the refracted ray $\mathrm{BA}$, can be represented by $C B+2 B A$. Following a similar reasoning, the resistance along the straight line CDA can be represented by $C D+2 D A$. Although the path $C B+B A$ is greater than the path CDA, therefore, the sum of the resistances $C B+2 B A$ is smaller than the sum of the resistances $C D+2 D A$ for a certain position of $\mathrm{B}$. As such, assuming that light follows the easiest path, the problem of refraction is reduced in this case to a problem of geometric nature, which Fermat put in the following terms: given 
two points $\mathrm{C}$ and $\mathrm{A}$, find the point of refraction $\mathrm{B}$ such that the sum of the resistances $C B+2 B A$ is a minimum.

By tackling this problem, Fermat presented a demonstration, based on a numeric example, that the straight line was not necessarily the most economical path for the refraction of light. His strategy was to apply his "maxima and minima method" to interpret the principle of the easiest path as a principle of least time.

I arrived at that without effort, but the investigation had to be carried farther; for, in order to satisfy my principle, it is not sufficient to have found a certain point $\mathrm{F}$ through which the natural movement is performed more quickly, more easily and in less time than along the straight line COG, but it was necessary to find the point through which the movement would be made in less time than through any other point taken on either side. For this purpose I had to appeal to my method of maxima and minima which expedites the solution of this sort of problem with much success. 11, p. 460]

Initially Fermat assumed that through his method of maxima and minima, the principle of the simplest path would lead to a different law than the one proposed by Descartes, in which he didn't believe [12, p.356], 13 . pp.457-8], [10, pp.485-6]. When informed that the available empirical data was in agreement with the relation proposed by Descartes, however, he chose to follow a different path, considering the existence of an infinite number of possible proportions which, although different from the true one, would approximate it in such a way as to deceive even the most capable observer [8, p.142]. Forced in a sense to not completely consider the relation proposed by Descartes, Fermat therefore considered that the true relationship could be such that it was very close to, although not identical to this relation.

But how to identify it? It is here that Fermat's thinking regarding the use of abstract mathematical principles as the heuristic foundation of his work becomes clear. As well pointed out by Sabra, the question of the truth or falsehood of the law of refraction was not decided, in Fermat's mind, solely by experimentation It had to be based on a foundation that Fermat considered to be more solid, because then it would rest on assumptions whose truth he considered beyond any doubt [8, p.143].

To finish his calculations, Fermat saw himself faced with a result that he [mistakenly] considered to be identical to the one obtained by Descartes as evidence of his law of sines 20 feeling extremely surprised that such a result had been obtained by Descartes from an assumption that the speed of light in denser media was greater than

\footnotetext{
${ }^{20}$ In his Synthèse Pour les Réfractions, Fermat attributed to Descartes a theorem based on the relation $\frac{\sin i}{\sin r}=\frac{v_{i}}{v_{r}}=n$. The correct relation was $\frac{\sin i}{\sin r}=\frac{v_{r}}{v_{i}}=n .8$ p.149]
}

in more rarefied media 21 - as opposed to his assumption. After all, he wondered, how could it be possible to reach the same truth by diametrically opposed paths without resorting to paralogisms? 13, p. 152] Although both results confirm the constancy of the ratio between the sine of the angle of incidence and the sine of the angle of refraction, the ratio between the speeds of incidence and refraction is reversed.

Fermat ended up conceding that Descartes had made an important discovery, of an empirical nature, but would claim the evidence, the effective demonstration of what he considered to be an important truth, for himself 13 , p.142].

In his exchange with Clerselier 22 initially mediated through his correspondence with La Chambre, he sought to clarify his point of view. Clerselier admitted that Fermat's proof, although logically valid, was only a moral and not a physical principle, and as such it wasn't or couldn't be the cause of any natural effect. His argument was that it wasn't this principle that made nature act, but a force that resides in all things and the disposition of these to receive it, which works without prediction, without choice and through a 'required determination' [12, p.465]. In his reply to Clerselier, Fermat wrote:

I believe that I have often said both to M. de la Chambre and you that I do not pretend, nor have I ever pretended to be in the inner confidence of Nature. She has obscure and hidden ways which I have never undertaken to penetrate. I would have only offered her a little geometrical aid on the subject of refraction, should she have been in need of it. But since you assure me, Sir, that she can manage her affairs without it, and that she is content to follow the way that has been prescibed to her by M. Descartes, I willingly hand over to you my alleged conquest of physics; and I am satisfied that you allow me to keep my geometrical problem - pure and in abstracto, by means of which one can find the path of a thing moving through two different media and seeking to complete its movement as soo as it can [12, p. 483].

This answer with a clear tone of irony reveals Fermat's strategic choice to not prolong the discussion, since the doesn't find any receptivity to his ideas in the interlocutor. Contrary to what a hasty interpretation of his response may lead us to believe, he remained aware that his task was not to restrict the law of refraction to no more than a mathematical hypothesis, purely geometric, in abstracto,

\footnotetext{
21 This hypothesis was deeply troubling to Fermat, who considered that light should have a finite velocity and move slower in denser media. In the mid 19th century, with the experiments of Foucault and Fizeau to measure the speed of light, the issue would be decided in favor of Fermat.

${ }^{22}$ Claude Clerselier (1614-1684) was a French publisher who edited and translated several of Descartes' works, especially his letters.
} 
but that its demonstration as a true principle of physics would vindicate an approach based on the principle of least time.

\section{Mechanisms and mathematical principles: a dynamic convergence of representation}

The works of Descartes and Fermat presented above were presented to clarify two different theoretical approaches to physical phenomena, more specifically to the phenomenon of the refraction of light - characterized respectively by the use of heuristic strategies based on mechanisms and mathematical principles.

Working on a framework that sought to remove the final Aristotelian causes from the analysis of physical phenomena, Descartes and his followers discarded a priori any attempt to assign purpose to physical phenomena, categorizing them as metaphysical. In this sense, the use of analogies and mechanisms, such as a ball hitting the water surface as a representation of the passage of light through media with different refrangibilities, gave him causal explanations for the phenomena of light reflection and refraction.

In contrast to this perspective and making use of his principle of least time, a clearly teleological principle, Fermat advocates the legitimacy and authority of the use of mathematical principles to carry out logical demonstrations of empirical laws.

In Pierre Duhem, we found a characterization of the physical theories that can help us understand Fermat's perspective. According to Duhem, a theory of physics is a system of mathematical propositions, deducted from a small number of principles, that seeks to represent a set of experimental laws in the most simple, complete and accurate way possible [1, p.19]. In this sense, Duhem redefines the objective and the nature of physical theory not in terms of explanation, that is, an understanding of the real causes of the phenomena - as did Descartes - but as a representation (or as linguistic symbols) that have no intrinsic connection with the properties that they represent, providing only the relation of the sign to the thing signified. [1, p.20]

In mechanical theories, on the other hand, all the physical quantities on which the laws rest, in the condition of being composed of geometric and mechanical elements of a certain material system, are imposed, conditioning all hypotheses to the implications of the dynamic properties of this system. This is the case, for example, of the particles in motion, used by Descartes in his explanatory mechanisms for the propagation of light [14, p.154-155].

Taking as reference Duhem's critical analysis regarding what he characterized as two methods of construction of physical theories - the synthetic method and the analytical method, each providing different visions about the meaning and objectives of theories [15, p.95] - we can expand our comprehension of the mechanisms and mathematical principles as non-exclusive, but dynamically converging heuristic strategies of representation.

The synthetic method - which is based on the Cartesian posture - consists in explaining physical phenomena through the construction of mechanical models, which are mechanisms made up of concrete elements, such as masses in motion. The analytical method is geared toward a more abstract approach, in the axiomatic form, generally appealing to principles of extremes (maxima and minima) - as is clearly the case of Fermat with his principle of least time, and later Maupertuis with his principle of least action, formulated strictly through concepts and propositions following the protocols of the geometrical model.

An example of this possible convergence of heuristic strategies can be found in Descartes himself who, recognizing that the once the mind can't capture, through logical propositions, the essential relations that exist in the world (metaphysical certainty), admits the provisional use of hypothetical relations between the theoretical knowledge base and the propositions that one wants to demonstrate (moral certainty) 6, rule VIII], [16, p.590]. Descartes and his interlocutors were trying to demonstrate the existence of an (anaclastic) curve in which parallel rays, refracted by a given surface, converge to a single point. For this explanation, Descartes made use of the geometrical proportions of the hyperbole, articulating mathematical reasonings (mathematical principles) and the physical demonstration of the curve (mechanisms) 17, pp.141-142].

Another example, also from Descartes, can be extracted from another one of his Discourses on the Method, Les Météoros, where he presents a quantitative determination of the rainbow phenomenon using both the synthetic method - through his model of the spherical drop and his explanation for the colors using the rotation of the particles of light, modeled as rotating spheres [2, pp.332333 ] - as the analytical method - which according to the observed facts, enabled the geometric modeling for the determination of primary and secondary arcs and angles capable of producing the phenomenon [2, pp.337-344].

Modeling the water droplets in suspension in the atmosphere as a spherical drop and considering the sun rays hitting it as straight parallel lines, the position of arches was explained, following the outline of Figure 9

For a continuous variation of the incident rays, the values of the spread out rays, determined from the geometrical model used by Descartes, ranged between $40^{\circ}$ and $42^{\circ}$ for the primary arc and $50^{\circ}$ and $52^{\circ}$ for the secondary arc [2, p.336], indicating that the luminous rays that define the rainbow are those whose deviations obey what we could call a principle of least angle. He also appealed to an analogy between the colors produced by the passage of light through the spherical water drop and those arising from the dispersion of light by a prism, which also depend on a minimum angle for their oc- 


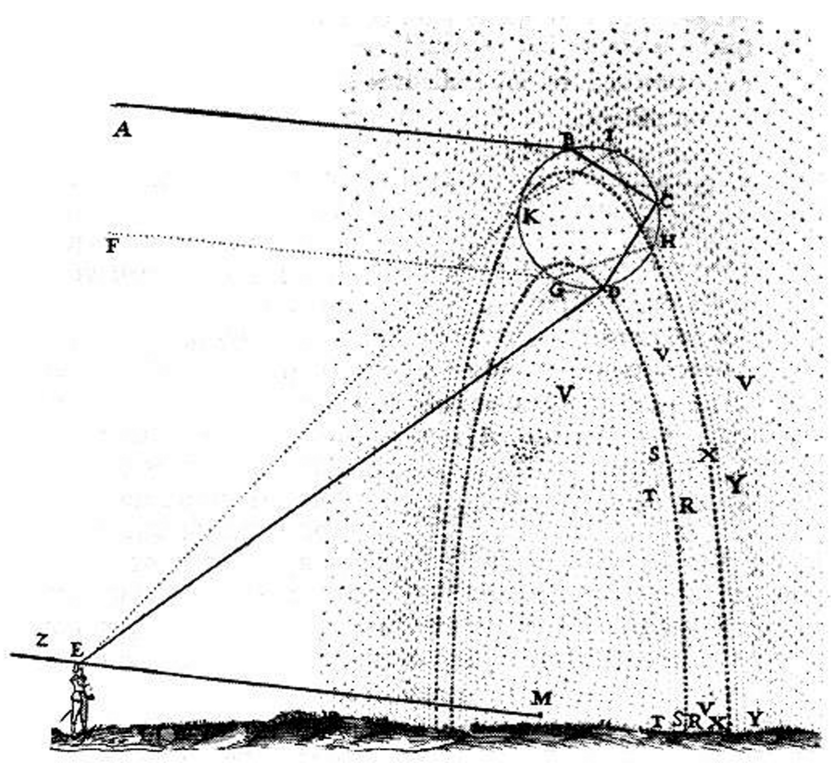

Figure 9: Drawing by Descartes explaining how a drop of water decomposes light to form the rainbow. Source: ref. [2 p. 328].

currence [2, p.341]. In Figure 10 the sketches used by Descartes to illustrate this analogy are shown.

In Fermat we can also recognize signs where the presence of mechanisms was accepted, although he made clear that these were dependent on principles capable of performing what he characterized as 'effective demonstration' 13 p.142]. To make use of his maxima and minima method in his demonstration of the law of refraction - a demonstration based on abstract principles, as we saw earlier - he was able to reconcile the existence of empirical data that presented itself in defense of Descartes 23

The original idea, which had served as the starting point for the principle of Fermat, was subsequently taken up by Pierre-Louis Moreau de Maupertuis (1698-1759) in 1744, who offered a new interpretation of the principle of economy, arguing that instead of traveling shorter distances or times, light took a path that minimized what he described as 'quantity of action' ([21, p.262], [22, p.456], 23, p.174]). In a certain sense, Maupertuis' strategy sought to circumvent the constraints generated by the exaggerated associated metaphysical dimension,

\footnotetext{
${ }^{23}$ Two authors who, in the second half of the 19th century, also made use of convergent heuristics strategies - combining mechanisms and mathematical principles - in the search for a statistical reasoning for the general laws of Thermodynamics were J. C. Maxwell (183179) and L. Boltzmann (1844-1906). With Boltzmann, the use of mechanical analytical tools (based on mathematical principles), guided by his atomistic perspective on the structure of matter (mechanisms), enabled him to treat microscopic motion mathematically and, subsequently, to construct a statistical approach as heuristic representational resource to understand relations between mechanics and thermodynamics within his research program. In Maxwell, we find not only the combination of his gas model ("hard sphere model") with the existence of a "function distribution of molecular speeds", but also his subsequent use of the Lagrangian and Hamiltonian formulation of analytical mechanics in the theoretical organization of electromagnetism to reconcile his mechanical interpretation. 18, 19, 20, p. 297].
}

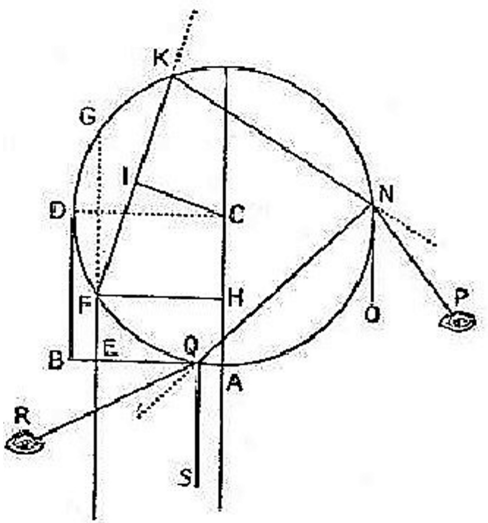

(a) Separation of sunlight in a drop of water

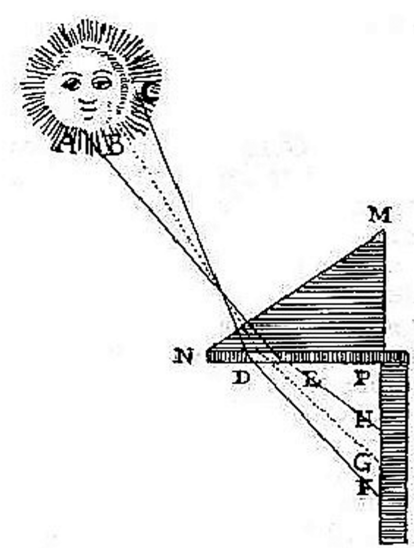

(b) Separation of sunlight in a crystal prism

Figure 10: Sketches used by Descartes to illustrate his analogy. See ref. [2 p.341]

both by the idea of minimizing the path of light as by minimizing the travel time.

Meditating deeply on this subject, I thought whether light, which abandons the shortest path (or the straight line) when it passes from one medium to another, could also fail to follow the fastest path. Indeed, what preference should time have over space here? As light could no longer follow, at the same time, the shortest and the fastest route, why would it take one and not the other? In fact, it follows neither, it takes a path that has a more real benefit: the path it takes is the one in which the quantity of action is minimal 24,24

Maupertuis' reasoning was that when a body is carried from one point to another, a certain "action" is required. Considering that it depends on both the speed of the body and on its path - combined, and not on each one

${ }^{24}$ An important translation (in Portuguese) of Maupertuis's article can be found in Ref. 23, along with a collection of his life and work, as well as an abbreviated historical description of the various types of variational principles used in Physics from Fermat (17th century) to the 20th century. 
separately - he defined it as being proportionate to the sum of the paths multiplied by their corresponding speeds. In this sense the "quantity of action" will be greater as the speeds and paths taken increase, and it's exactly in this that the true cost of nature lies, and it's in this, therefore, where savings are maximized in the movement of light 24 .

According to Sabra, Maupertuis' procedure was no less metaphysical than the Fermat's, being as or more arbitrary than it in so far as Fermat still could argue that time was a basic and natural concept, whose intimate relationship with motion made it a viable candidate as a minimum quantity, while Maupertuis' "action" was artificially constructed [8, p. 157].

For Martins and Silva, Maupertuis was clearly aware of the historical precedents of his principle and of the metaphysical roots of his ideas - already present in the works of Aristotle and some scholastic thinkers - and did not consider these philosophical influences inappropriate. 22, p.462].

It was with the work of Euler (1744) and Lagrange (1867), the latter one expanding the applications of the "principle of least action" made by the first, that Maupertuis' principle (as a principle of extremes) consolidated itself - no longer as a metaphysical principle, but as a result of mechanical laws and as a heuristic resource for the solution of different problems of dynamics [25, p.43-82]. With Lagrange, a new representation of mechanics was born, called Analytical Mechanics, that had an important impact on all physics 26. Subsequently, working on the rationalization of geometrical optics and inspired by Lagrange's representation, Hamilton (1931) added new representational heuristic elements to these two fields, in addition to articulating a new representation for Analytical Mechanics.

From the elements gathered here, a working hypothesis could consist in understanding the mechanisms and mathematical principles as different forms of representation of natural phenomena in a converging dynamic for the comprehension of physical theories. In this sense, the first should reveal the functions and the dynamics of the causal relationships involved in a given phenomenon - which is of great relevance for its reproduction and for the application of its empirical laws; and the second should serve as guide and abstract modeling, in addition to formalizing these laws. From the mechanisms, we expect an indication of the possibilities to reconstruct the conditions of nature, while the mathematical principles should point to its modus operandi, for example, how it operates according to the principles of economy (or optimization) - as is the case with the principle of minima.

From the heuristic point of view, we raise the possibility of understanding both representations as complementary, using the thesis that it's up to the mechanisms to capture the constituting material principle of the phenomenon, while the abstract mathematical principles should take care of its formal organization.

We believe that the exploitation of the compatibility and complementarity between these two representations may also help us understand both the construction dynamics of scientific theories and the models of rationalization and methodologies associated with them.

\section{Implications for physics education}

According to Hodson, a central aspect of scientific literacy for the 21st century is directly related to what he characterizes as "learning about science", that is, an understanding of its nature and methods, an appreciation of its history and development, and an awareness of the complex social relations that constitute it ([27, p.23], 28, p.15]). In this direction, approaches involving the history and philosophy of science have been pointed out as relevant $([29],[30],[31],[32]$, despite the immense challenges to their effective incorporation into curricula 33 .

By focusing on the use of mechanisms and mathematical principles as representational heuristics in the physics of Descartes and Fermat, the analysis developed in sections II, III and IV can help teachers and students to better understand the relevant historical and epistemological aspects inherent in the dynamics of the construction of scientific theories. These elements, in addition to being essential for an understanding of the nature of science (NoS), may broaden our pedagogical tools in the treatment of refraction phenomena. In addition, to understand causal explanations (in this case associated with the use of mechanisms) and abstract representations (inherent mathematical principles) as legitimate, complementary and convergent heuristic representational resources in the formulation of physics's principles and laws can help to relativize attitudes and beliefs about an absolute and restrictive view of the "scientific method", still deeply rooted in school culture.

In recent years, the relationships between learning and representations have been studied in the physics teaching, strongly suggesting that the representations we use in physics play a critical role in the effectiveness of students' learning, engaging them interactively in their learning environments and contributing significantly in both conceptual understanding and broadening their problem-solving strategies in this field $([34,35], 36]$, [37], [38], 39], [40], [43], [41], 42], [44], 46]).

Highlighting the importance of an adequate understanding of the thought processes underlying the production of knowledge in physics teaching, Frederick Reif establishes as a basic premise of his work the following idea:

One cannot teach physics effectively without an adequate understanding of the thought process need in this field (no more than one can teach someone how to play good chess 
without an adequate understanding of the thought process need to play that game) 35 p.17].

It's precisely here that the different representations of physical theories - such as those in the work of Descartes and Fermat presented in this paper - show their pedagogical effectiveness, in so far as they explicit the thought processes of these authors and incorporate different perspectives and communication potential ("affordances" [47]) around the topic studied. Once understood, such thought processes can play a relevant role in the teaching and learning strategies. Since one of the basic elements in the teaching of science should be the heuristic structure proper to the production of knowledge in science.

Considering that the affordances of different representations determine the role they can play in communication, and thus in the sharing of knowledge, Fredlung et al sought to explore what they characterize as disciplinary affordances of representation that is the inherent potential of that representation to provide access to disciplinary knowledge. In this sense, they argue that "physics learning involves coming to appreciate the disciplinary affordances of representations" 44].

An interesting way of developing these ideas in the school context is to make use of "case studies". According to Stinner et al "case studies are historical contexts with one unifying idea, designed according to the guidelines for writing a large context problem" 45, p. 620]. Although it was not the purpose of this article to develop a historical case study with didactic purposes, which would require an approach with aspects other than those presented here, we believe we have gathered elements that can subsidize works in this direction.

A central aspect of the analysis conducted here and illustrated with elements drawn from the physics of Descartes and Fermat is that Representation is a heuristic tool for building laws, principles, and physical theories.

\section{Conclusions}

Throughout this article, we have sought to demonstrate that the use of mechanisms and mathematical principles were the representational structure of the physical phenomena used by Descartes and Fermat, respectively, in their investigations in Physics. With examples taken from the works of these authors, specifically in their analysis of the light refraction phenomenon, we revealed their distinct commitments and conceptions, thought processes, which we don't consider to be mutually exclusive - even if we recognize their different functions - for the representation of physical phenomena. From the heuristic point of view, we advocate that both approaches can be seen as complementary and convergent, using the thesis that it's up to the mechanisms to capture the constituting material principle of the phenomenon, and up to the abstract mathematical principles to reveal their formal organization. By emphasizing aspects related to the dynamics of the construction and development of scientific theories, heuristic elements essential to an understanding of nature of science and therefore to teaching and learning physics was indicate.

\section{Acknowledgements}

The authors thank the Institute of Physics of the University of Brasília, the Department of Philosophy and the Department of Economics of University of São Paulo for their support. We are also grateful to the referees whose observations and suggestions helped to improve this work.

\section{References}

[1] Pierre Duhem, The Aim and Structure of Physical Theory (Princeton University Press, Oxford, 1954).

[2] René Descartes, in: Oeuvres de Descartes, edited by Charles Adam and Paul Tannery (Vrin, Paris, 1996), 11 v., v. 6. English translation of La Dioptrique by J. Cottinghan, R. Stoothoff and D. Murdoch, in The Philosophical Writings of Descartes (Cambridge University Press, Cambridge, 1985), v. 1.

[3] Mario Bunge, Philosophy of the Social Science 27, 410 (1977).

[4] Imre Lakatos, in The Methodology of Scientific Research Programmes, edited by J. Worral and G. Currie (Cambridge University Press, Cambridge, 1984), v. 1.

[5] J.R.N. Chiappin, Duhem's Theory of Science: The Interplay Between Philosophy and History of Science. PhD. Thesis, Department of Philosophy, University of Pittsburgh, 1989.

[6] René Descartes, in: Oeuvres de Descartes, edited by Charles Adam and Paul Tannery (Vrin, Paris, 1996), v. 10. English translation of La Dioptrique by J. Cottinghan, R. Stoothoff and D. Murdoch, in The Philosophical Writings of Descartes (Cambridge University Press, Cambridge, 1985), v. 1.

[7] Pierre Duhem, Revue des Questions Scientifiques 40, 463 (1896).

[8] A.I. Sabra, Theories of Light (Cambridge University Press, Cambridge, 1981).

[9] Pierre de Fermat, in: Oeuvres de Fermat (Correspondance), edited by Paul Tannery and Chales Henry (Gauthier-Villars et Fils, Paris, 1996), 4 v., v. 2, p. 106112.

[10] Pierre de Fermat, in: Oeuvres de Fermat (Correspondance), edited by Paul Tannery and Chales Henry (Gauthier-Villars et Fils, Paris, 1996), 4 v., v. 2, p. 485496.

[11] Marin Cureau de la Chambre, La Lumière (Chez Iacqves D‘Allin, Paris, 1657).

[12] Pierre de Fermat, in: Oeuvres de Fermat (Correspondance), edited by Paul Tannery and Chales Henry (Gauthier-Villars et Fils, Paris, 1996), 4 v., v. 2, p. 354359.

[13] Pierre de Fermat, in: Oeuvres de Fermat (Correspondance), edited by Paul Tannery and Chales Henry 
(Gauthier-Villars et Fils, Paris, 1996), 4 v., v. 2, p. 457463.

[14] Pierre Duhem, Revue des Questions Scientifiques 31, 139 (1899).

[15] Pierre Duhem, L'Èvolution de la Mécanique (A. Joanin, Paris, 1980). German translation, P. Frank, Die Wandlungen der Mechanik und der mechanischen Naturerklärung (J.A. Barth, Leipzig, 1912). English translation, Michael Cole, The Evolution of Mechanics, Alphen aan den Rijn (Sijthooff and Noordhoff, Netherlands, 1980).

[16] J.R.N. Chiappin and A.C. Leister, Princípios-Revista de Filosofia 20, 583 (1977).

[17] René Descartes, Oeuvres de Descartes (Correspondance), edited by Charles Adam and Paul Tannery (Vrin, Paris, 1996), v. 10.

[18] C.C. Laranjeiras and J.R.N. Chiappin, A Heurística de Boltzmann e a Emergência do Programa MecânicoEstatístico, Revista Brasileira de Ensino de Física 28, 297 (2006), DOI: http://dx.doi.org/10.1590/S180611172006000300006 .

[19] C.C. Laranjeiras and J.R.N. Chiappin, A Construção de uma Teoria de Ensembles: Antecedentes em Maxwell e Boltzmann, Revista Brasileira de Ensino de Física 30, 1601 (2008), DOI: doi:http://dx.doi.org/10.1590/ S1806-11172008000100015.

[20] J.R.N. Chiappin, Cognitio 15, 289 (2014).

[21] René Dugas, Histoire de la Mécanique (Neuchâtel, Paris, 1950).

[22] Roberto de Andrade Martins and Ana Paula Bispo da Silva, Maupertuis, d'Arcy, d'Alembert e o princípio de ação mínima na óptica: uma análise crítica, Revista Brasileira de Ensino de Física 29, 455 (2007), DOI: doi:http://dx.doi.org/10.1590/S010247442007000300017.

[23] Ildeu de Castro Moreira, Revista Brasileira de Ensino de Física 21, 172 (1999).

[24] P.L. de Maupertuis, Accord des Différentes Lois de la Nature qui Avaient Jusqu'ici paru Incompatibles (Mémoires de l'Académie des Sciences de Paris, Paris, 1744).

[25] Jojomar L. da Silva Abordagens Comparativas de Ciclos e de Potenciais da Termodinämica: Escolha Racional ou Pragmática?. Tese de Doutorado, Departmento de Filosofia, Universidade de São Paulo, 2015.

[26] Mario Bunge, American Journal of Physics 25, 211 (1957).

[27] Derek Hodson, Towards scientific literacy (Sense Publishers, Rotterdam, 2008).

[28] Derek Hodson, Teaching and Learning about Science Language, Theories, Methods, History, Traditions and Values (Sense Publishers, Rotterdam, 2009).

[29] J. Solomon, J. Duveen, L. Scot and S. McCarthy, Journal of Research in Science Teaching 29, 409 (1992).

[30] W.F. McComas (ed), The Nature of Science in Science Education Rationales and Strategies (Kluwer Academic Publishers, The Netherlands, 2000).

[31] A.R. Irwin, Science Education 84, 5 (2000).

[32] Huann-Shyang Lin and Chung-Chih Chen, Journal of Research in Science Teaching 99, 773 (2002).

[33] Dietmar Höttecke and Cibelle Celestino Silva, Science and Education 20, 293 (2011).

[34] A. Van Heuvelen, American Journal of Physics 59, 891 (1991).
[35] Frederick Reif, Journal of Science Education and Technology 4, 261 (1995), DOI: 10.1007/BF02211259.

[36] L.C. McDermott, American Journal of Physics 71, 1127 (2001), DOI: http://dx.doi.org/10.1119/1.1389280

[37] A. Van Heuvelen and X. Zou, American Journal of Physics 69, 184 (2001), DOI: http://dx.doi.org/10 $1119 / 1.1286662$.

[38] D. Hestenes, American Journal of Physics 71, 104 (2002), DOI: http://dx.doi.org/10.1119/1.1522700.

[39] P.B. Kohl and N.D. Finkelstein, Physical Review Physics Education Research 2, 010102 (2006), DOI: https:// doi.org/10.1103/PhysRevSTPER.2.010102

[40] P.B. Kohl, D. Rosengrant and N.D. Finkelstein, Physical Review Physics Education Research 3, 010108 (2007), DOI: https://doi.org/10.1103/PhysRevSTPER. 3.010108

[41] John Airey and Cedric Linder, Journal of Research in Science Teaching 46, 27 (2009), DOI: 10.1002/tea.2065.

[42] Kok-Sing, Seng Chee Tan and Jennifer Yeo, Journal of Research in Science Teaching 33, 1775 (2011), DOI: http://dx.doi.org/10.1080/09500693.2010.508899.

[43] David Rosengrant, Alan Van Heuvelen and Eugenia Etkina, Physical Review Physics Education Research 5, 010108 (2009), DOI: https://doi.org/10.1103/ PhysRevSTPER .5.010108

[44] Tobias Fredlund, John Airey and Cedric Linder, American Journal of Physics 33, 657 (2012), DOI: 10.1088/ 0143-0807/33/3/657

[45] Arthur Stinner, Barbara A. Mcmillan, Don Metz, Jana M. Jilek and Stephen Klassen, Science and Education 12, 617 (2003). DOI: https://link.springer.com/ article/10.1023/A:1025648616350.

[46] Tobias Fredlund, John Airey and Cedric Linder, American Journal of Physics 36, 05001 (2015), DOI: 10.1088/ 0143-0807/36/5/055001.

[47] J.J. Gibson, The Ecological Approach to Visual Research (Houghton Mifflin, Boston, 1979). 